\section{Prophylactic Donor Lymphocyte Infusion to Prevent Relapse after Allogeneic Stem Cell Transplantation}

Keywords: Donor lymphocyte infusion; Acute myeloid leukemia; Allogeneic stem cell transplantation; Graft versus leukemia effect; Graft versus host disease

\begin{abstract}
Donor lymphocyte infusion (DU) has been used to treat disease relapse after stem cell transplantation, but high incidence of graftversus-host disease (GVHD) after traditional DU, and low response rates with robust relapse in acute leukemia have limited its wide application. Nonetheless, the promising graft versus leukemia effect elicited by DU makes it a viable option, especially in the state of minimal residual disease after stem cell transplantation. Prophylactic donor lymphocyte infusion has been explored as a more tolerable, less toxic and efficacious option to prevent relapse. This review will summarize the results of the latest prophylactic DU studies, and discuss our ongoing prophylactic DLI clinical trial after T cell depleted stem cell transplantation in patients with high risk hematologic malignancies.
\end{abstract}

\section{Introduction}

Hematopoietic stem cell transplantation (SCT) is a potentially curative treatment for patients with hematologic malignancies. There has been tremendous progress in the past several decades in allogeneic SCT with better outcomes through improvements in supportive care, expansion of stem cell donor options (HLA-matched unrelated donors (MUD), haploidentical related donors, and cord blood units (CBUs) et al), and introduction of better tolerated reduced intensity conditioning (RIC) regimens [1,2]. T-cell depletion is another approach that enhances tolerability by reducing acute and chronic graft-versus-host disease (GVHD), which results in tremendous transplantation related morbidity and mortality. We have employed in vivo T-cell depletion (TCD) with alemtuzumab for over 10 years at the University of Chicago and confirmed lower rates of acute and chronic GVHD and similar overall survival when comparing to regimens without $\mathrm{T}$ cell depletion [3]. However, relapse remains highly problematic after transplant [4], and T-cell depletion further increases rate of relapse. There has been relatively little progress in reducing the incidence of relapse following allogeneic SCT. T-cell depletion may enable patients to successfully undergo transplant with low incidence of GVHD, setting the platform to allow approaches that harness the immune system to prevent relapse after transplant.

\section{Donor lymphocyte infusion for relapse after SCT}

Donor lymphocyte infusion (DLI) has been shown to induce remissions post-transplant in patients with relapsed hematologic malignancies, most consistently in patients with CML, and to a lesser extent in AML, multiple myeloma and myelodysplasia [5]. The major complications with DLI are the development of GVHD and cytopenias. Acute GVHD develops in up to $40-60 \%$ of patients who receive DLI. However, the development of GVHD does not always correlate with graft versus leukemia (GVL) activity [6].
Journal of

Clinical \& Medical Case Reports

\section{Hongtao Liu*}

Section of Hematology/Oncology, University of Chicago, Chicago, IL, USA

\section{Address for Correspondence}

Hongtao Liu, MD, Section of Hematology/Oncology, University of Chicago 5841 South Maryland Avenue, MC 2115 Chicago, IL 60637, USA. Tel: (773) 834-0589; Fax: (773) 702-3163; E-mail: hliu2@medicine. bsd.uchicago.edu

Submission: 24 April 2013

Accepted: 29 May 2013

Published: 30 May 2013

\section{Prophylactic DLI after SCT}

Since DLI seems to be most effective for patients with minimal residual disease, the role of prophylactic DLI (pDLI) for high risk patients after stem cell transplant has been explored, both in the myeloablative conditioning setting [7-12] and in the RIC setting [1318].

In the myeloablative setting, the largest series was reported by Montero et al. [12]. One hundred thirty-eight patients with hematologic malignancies received myeloablative $\mathrm{T}$ cell-depleted peripheral blood stem cell transplant from an HLA-identical sibling donor. 112 patients with less than Grade II acute GVHD received 1 or 2 pDLI (T-cell add back) at $10-50 \times 10^{6} \mathrm{CD} 3+$ cells $/ \mathrm{kg}$ between days 45 and 100 post SCT. Overall survival (OS), relapse-free survival (RFS), relapse, and transplant-related mortality (TRM) were $58 \%, 46 \%, 40 \%$, and $20 \%$, respectively, after a median follow-up of 4 years. Fifty-three (39\%) and 21 (15\%) patients developed Grade II-IV and III-IV acute GVHD respectively. Forty-two (36\%) had limited and 29 (25\%) had extensive chronic GVHD. These rates of chronic GVHD are similar compared with that of T cell-replete SCT. However, it has been noted that chronic GVHD after T cell add back may be associated with less mortality and relapse, at least in standard-risk patients [12].

Summarizing several studies to date, the risk of relapse after pDLI with myeloablative conditioning has ranged from 18 to $69 \%$ with TRM ranging $6-52 \%$. This translates into leukemia free survival (LFS) of more than $40 \%$ at 2 years after SCT [7-12]. Schaap's study [10] compared outcomes of patients receiving DLI with those patients not receiving DLI, and demonstrated lower relapse rates resulting in improved LFS in those receiving DLI. Furthermore, the incidence of acute and chronic GVHD and the risk of TRM did not seem to differ from the expected outcomes after conventional transplant without DLI.

Various studies have analyzed outcomes of pDLI after RIC [1318]. Barge et al. [13] reported on 11 patients, who were given planned DLI at 6 months after RIC MRD SCT with in vitro TCD. The DLI dose, given as unselected mononuclear cells, was based on disease status. Patients with relapse or progression at 6 months received 10 $100 \times 10^{6} \mathrm{MNC} / \mathrm{kg}$ plus IFN- $\alpha$ compared with only $10 \times 10^{6} \mathrm{MNC} / \mathrm{kg}$ for patients with stable disease or mixed chimerism. In the 11 patients receiving DLI, 5 responded $(\mathrm{CR}=3 ; \mathrm{PR}=2)$ and 1 patient had stable disease. Acute GVHD developed in six patients, chronic GVHD in four patients and GVHD accounted for the death of one patient. 
Schmid et al. [16] studied 75 patients with high-risk AML or MDS who received non-myeloablative conditioning with fludarabine, cytarabine and low-dose total body irradiation. Patients were scheduled to receive prophylactic DLI after 120 days if there was no evidence of GVHD and they were off immunosuppression medications. Of the 75 patients enrolled, only 12 patients were able to receive prophylactic DLI due to early relapse, GVHD and other transplant-related complications in the others, demonstrating the limitations of this approach. Interestingly, only 2 out of the 12 patients who received pDLI had disease relapse during the follow-up, with an impressive LFS of $92 \%$ at 3 years for this population after transplantation [16].

Two recent publications provide additional support to the feasibility and efficacy of pDLI after TCD allo-SCT $[19,20]$. In a prospective study, Liga et al. [19] reported on 15 out of 56 consecutive patients who underwent allo-SCT with an alemtuzumab-containing regimen (10 to $20 \mathrm{mg}$ ) and received prophylactic DLI at a median of 162 days post-transplantation. Seven received standard myeloablative conditioning while eight received reduced-toxicity myeloablative conditioning for age $>55$ years old or significant co-morbidities. Six of the 8 patients $(75 \%)$ who received pDLI with mixed chimerism converted to stable, complete donor chimerism and none of the patients who received pDLI relapsed. $47 \%$ of DLI recipients developed Grade III-IV GVHD and all four deaths were due to GVHD-related causes. This suggests that while low-dose pDLI after conditioning with alemtuzumab could decrease the risk of relapse; this approach is associated with a relatively high incidence of severe GVHD.

On the other hand, Krishnamurphy et al reported promising results of pDLI in AML and MDS patient in a retrospective study [20]. They evaluated the efficacy and toxicity of pDLI in 62 patients after TCD (alemtuzumab at $100 \mathrm{mg}$ or ATG at $6 \mathrm{mg} / \mathrm{kg}$ ) RIC alloSCT for AML or MDS. Despite 52\% patients received pDLI within 6 months post-SCT, the 5 -year incidence of GVHD was only $31 \%$. The overall survival of $80 \%$ and event-free survival of $65 \%$ at 5 years in the recipients of pDLI supports the use of pDLI to safely promote durable remission after TCD RIC allo-SCT for AML or MDS patients.

\section{Rationale for repetitive DLI}

A recent study demonstrated that patients with AML or CML in remission following SCT exhibited significant numbers of CD8+ $\mathrm{T}$ cells that recognize epitopes derived from leukemia-associated antigens. However, these cells failed to proliferate, release cytokines, or de-granulate in response to antigen-specific stimuli. The use of IL-15 or high-dose IL-2, elimination of CD4+ regulatory T cells, and blockade of PD-L all failed to rescue the activation of these CD8+ T cells in in vitro assays, as the mechanism for CD8+ unresponsiveness after SCT seemed to be replicative senescence [21].

Multiple sources of chronic stimulation following SCT may contribute to potential T-cell senescence, including GVHD, GVL activity, infection, persistent stimulation of $\mathrm{T}$ cells by residual leukemia cells, and slow reconstitution of CD4+ T cells after SCT during homeostatic proliferation to repopulate the T-cell pool. In addition, population dynamics of the $\mathrm{T}$-cell pool after transplant may be influenced by the intensity of the conditioning regimen before transplant, T-cell dose within the graft, immunosuppressive therapy, and the use of donor lymphocyte infusions [21]. Donor T cell tolerance or rapid senescence after allo-SCT may contribute to post-transplant relapse. Thus, preserving and maintaining a competent and reactive pool of CD8+ T-cell precursors after allo-SCT by repetitive DLI once a patient achieves remission, may prove efficacious in preventing relapse.

\section{Rationale to conduct prophylactic dose-escalation DLI to prevent relapse in hematologic malignancies after $T$ cell depleted allo-SCT}

Relapse remains a major issue in non-myeloablative $\mathrm{T}$ cell depleted allo-SCT, and DLI is a clinically available and established treatment that is most effective for minimal residual disease. Therefore, the role of dose-escalation prophylactic DLI for patients in remission to prevent relapse will be assessed in a pilot study. The study will focus on patients with high risk hematologic malignancies, including leukemia not in remission, lymphoma not achieving a partial response, or other diseases under poor control, undergoing allo-SCT. We reviewed the University of Chicago transplant database for patients with high-risk AML or MDS who underwent a T-cell depleted allogeneic transplant. Of the 145 patients with AML or MDS transplanted with active disease, relapse occurred at a median of 128 days (24 to 2364 days), with PFS of 137 days (4 to 3272 days) and OS of 214 days ( 4 to 3434 days). Patients had received either fludarabine/ melphalan/Alemtuzumab (flu/mel/campath) or clofarabine/ melphalan/Alemtuzumab (clo/mel/campath) conditioning regimens (unpublished data).

From our prior published data, after flu/mel/campath conditioning, patients with high-risk disease had a $39 \%$ probability of disease recurrence, a $39 \%$ probability of treatment-related mortality, and a $25 \%$ probability of progression-free survival at 1 year after stem cell transplant [22]. In our clo/mel/campath protocol database [23]. 35 out of 72 patients accrued had high risk, active disease, and had a 1 year PFS of $31 \%$ which is similar to the $25 \% 1$ year PFS seen with $\mathrm{flu} / \mathrm{mel} / \mathrm{campath}$. Additionally, the 1 year relapse rate was $29 \%$ for the whole cohort, making the 1 year relapse rate for the high risk patients comparable to 1 year relapse rate of $39 \%$ in the high risk patients after $\mathrm{flu} / \mathrm{mel} / \mathrm{campath}$. The cumulative probability of Grade II-IV acute GVHD was $33 \%$ in flu/mel/campath and $22 \%$ in clo/mel/campath study at 1 year, and 1 year TRM was $33 \%$ and $26 \%$, respectively $[22,23]$. Published data using fludarabine+bulsufan+alemtuzumab or ATG also has demonstrated comparable survival and relapse results in high risk hematologic malignancies $[24,25]$. However, this novel approach still requires feasibility testing of each step of the process, including enrollment of high-risk patients, early withdrawal of immune suppression and the ability to escalate DLIs. The clinical protocol has been approved by the IRB at the University of Chicago, and we are actively enrolling patients to the study at the present time.

In brief, fifty-six patients undergoing $\mathrm{T}$ cell depleted matched sibling (7/8 or $9 / 10$ matched related donor is allowed) or matched unrelated donor allogeneic stem cell transplant will be enrolled to the study. The immunosuppression will be tapered off at day 60 post alloSCT and eligible patients will receive dose escalation prophylactic donor infusion around day 75 to day 90 after allo-SCT. The starting dose of pDLI will be $2 \times 10^{5} / \mathrm{kg}$ (followed by $5 \times 10^{5} / \mathrm{kg} ; 1 \times 10^{6} / \mathrm{kg} ; 2 \mathrm{x}$ $10^{6} / \mathrm{kg} ; 5 \times 10^{6} / \mathrm{kg}$ ) for MRD; and $1 \times 10^{5} / \mathrm{kg}$ (followed by $2 \times 10^{5} / \mathrm{kg} ; 5 \mathrm{x}$ $10^{5} / \mathrm{kg} ; 1 \times 10^{6} / \mathrm{kg} ; 2 \times 10^{6} / \mathrm{kg}$ ) for MUD. Subsequent DLIs will be given in 4-8 week intervals. Acute GVHD is the most serious complication of donor lymphocyte infusion. Any patient who develops more than Grade II aGVHD will be taken off the study and be treated for acute GVHD. We will monitor for aGVHD regularly and have enlisted formal stopping rules. After every 10 patients have passed 6 months of follow up from immune suppression withdrawal, a cumulative incidence curve of aGVHD will be generated. The clinical trial has been posted at the Clinical Trials.gov website (NCT01839916). 
Citation: Liu H. Prophylactic Donor Lymphocyte Infusion to Prevent Relapse after Allogeneic Stem Cell Transplantation. J Clin Med Case Reports. 2013;1(1): 3.

There is another ongoing prophylactic DLI study after T cell depleted matched sibling stem cell transplant in the United Kingdom (NCT01240525). This randomized Phase II study will withdraw immunosuppression at day 40 post SCT, and patients are randomized to receive either single dose of $1 \times 10^{6} / \mathrm{kg}$ CD4 cells $/ \mathrm{kg}$ or no DLI between day 100 to 120 post-SCT. Our study differs in testing prophylactic DLI in both MRD and MUD SCT, starting at 5-10 folds lower T cell doses, with DLI dose escalation in order to minimize complications of GVHD while attempting to maximize the graft versus leukemia effect.

\section{Conclusion}

Relapse remains the major failure after allogeneic stem cell transplant and its prevention remains an unmet need. As various new strategies and novel treatments have been under exploration, DLI has proven efficacious to treat and prevent disease relapse after allo-SCT. The very low incidence of acute and chronic GVHD after in vivo $\mathrm{T}$ cell depletion provides an excellent platform to test different strategies of DLI to prevent relapse after allo-SCT with tolerable toxicities. Current studies in TCD allo-SCT may elucidate whether DLI-induced GVHD may be attenuated through decreased cell dose coupled with dose escalation administration and short term immunosuppression. Ultimately, this may translate into improvement in PFS and OS with good quality of life.

\section{References}

1. Gooley TA, Chien JW, Pergam SA, Hingorani S, Sorror ML, et al. (2010) Reduced mortality after allogeneic hematopoietic-cell transplantation. N Engl J Med 363: 2091-2101.

2. Sorror ML, Sandmaier BM, Storer BE, Franke GN, Laport GG, et al. (2011) Long-term outcomes among older patients following nonmyeloablative conditioning and allogeneic hematopoietic cell transplantation for advanced hematologic malignancies. JAMA 306: 1874-1883.

3. Van Besien K, Kunavakkam R, Rondon G, De Lima M, Artz A, et al. (2009) Fludarabine-melphalan conditioning for AML and MDS: alemtuzumab reduces acute and chronic GVHD without affecting long-term outcomes. Biol Blood Marrow Transplant 15: 610-617.

4. van den Brink MR, Porter DL, Giralt S, Lu SX, Jenq RR, et al. Relapse after allogeneic hematopoietic cell therapy. Biol Blood Marrow Transplant 16: S138-145.

5. Deol A, Lum LG (2010) Role of donor lymphocyte infusions in relapsed hematological malignancies after stem cell transplantation revisited. Cancer Treat Rev 36: 528-538.

6. Tomblyn M, Lazarus HM (2008) Donor lymphocyte infusions: the long and winding road: how should it be traveled? Bone Marrow Transplant 42: 569579 .

7. Barrett AJ, Mavroudis D, Tisdale J, Molldrem J, Clave E, et al. (1998) T celldepleted bone marrow transplantation and delayed $\mathrm{T}$ cell add-back to contro acute GVHD and conserve a graft-versus-leukemia effect. Bone Marrow Transplant 21: 543-551.

8. Ferrá C, Rodríguez-Luaces M, Gallardo D, Encuentra M, Martín-Henao GA et al. (2001) Individually adjusted prophylactic donor lymphocyte infusions after CD34-selected allogeneic peripheral blood stem cell transplantation. Bone Marrow Transplant 28: 963-968.

9. Nakamura R, Bahceci E, Read EJ, Leitman SF, Carter CS, et al. (2001) Transplant dose of CD34(+) and $\mathrm{CD} 3(+)$ cells predicts outcome in patients with haematological malignancies undergoing $\mathrm{T}$ cell-depleted peripheral blood stem cell transplants with delayed donor lymphocyte add-back. $\mathrm{Br} \mathrm{J}$ Haematol 115: 95-104.

10. Schaap N, Schattenberg A, Bar B, Preijers F, van de Wiel van Kemenade E, de Witte T (2001) Induction of graft-versus-leukemia to prevent relapse after partially lymphocyte-depleted allogeneic bone marrow transplantation by preemptive donor leukocyte infusions. Leukemia 15: 1339-1346.

11. Lee CK, de Magalhaes-Silverman M, Hohl RJ, Hayashi M, Buatti J, et al (2002) Prophylactic $T$ cell infusion after $T$ cell-depleted bone marrow transplantation in patients with refractory lymphoma. Bone Marrow Transplant 29: $615-620$
12. Montero A, Savani BN, Shenoy A, Read EJ, Carter CS, et al. (2006) T-cell depleted peripheral blood stem cell allotransplantation with T-cell add-back for patients with hematological malignancies: effect of chronic GVHD on outcome. Biol Blood Marrow Transplant 12: 1318-1325.

13. Barge RM, Osanto S, Marijt WA, Starrenburg CW, Fibbe WE, et al. (2003) Minimal GVHD following in-vitro $T$ cell-depleted allogeneic stem cell transplantation with reduced-intensity conditioning allowing subsequent infusions of donor lymphocytes in patients with hematological malignancies and solid tumors. Exp Hematol 31: 865-872.

14. de Lima $M$, Bonamino $M$, Vasconcelos $Z$, Colares $M$, Diamond $H$, et al (2001) Prophylactic donor lymphocyte infusions after moderately ablative chemotherapy and stem cell transplantation for hematological malignancies: high remission rate among poor prognosis patients at the expense of graftversus-host disease. Bone Marrow Transplant 27: 73-78.

15. Massenkeil G, Nagy M, Lawang M, Rosen O, Genvresse I, et al. (2003) Reduced intensity conditioning and prophylactic DLI can cure patients with high-risk acute leukaemias if complete donor chimerism can be achieved. Bone Marrow Transplant 31: 339-345.

16. Schmid C, Schleuning M, Ledderose G, Tischer J, Kolb HJ (2005) Sequential regimen of chemotherapy, reduced-intensity conditioning for allogeneic stemcell transplantation, and prophylactic donor lymphocyte transfusion in highrisk acute myeloid leukemia and myelodysplastic syndrome. J Clin Oncol 23: 5675-5687.

17. Meyer RG, Britten CM, Wehler D, Bender K, Hess G, et al. (2007) Prophylactic transfer of CD8-depleted donor lymphocytes after T-cell-depleted reducedintensity transplantation Blood 109: 374-382.

18. Dey BR, McAfee S, Colby C, Sackstein R, Saidman S, et al. (2003) Impact of prophylactic donor leukocyte infusions on mixed chimerism, graft-versushost disease, and antitumor response in patients with advanced hematologic malignancies treated with nonmyeloablative conditioning and allogeneic bone marrow transplantation. Biol Blood Marrow Transplant 9: 320-329.

19. Liga M, Triantafyllou E, Tiniakou M, Lambropoulou $P$, Karakantza M, et al. (2013) High alloreactivity of low-dose prophylactic donor lymphocyte infusion in patients with acute leukemia undergoing allogeneic hematopoietic cell transplantation with an alemtuzumab-containing conditioning regimen. Biol Blood Marrow Transplant 19: 75-81.

20. Krishnamurthy P, Potter VT, Barber LD, Kulasekararaj AG, Lim ZY, et al. (2013) Outcome of Donor Lymphocyte Infusion after T Cell-depleted Allogeneic Hematopoietic Stem Cell Transplantation for Acute Myelogenous Leukemia and Myelodysplastic Syndromes. Biol Blood Marrow Transplant 19: 562-568.

21. Beatty GL, Smith JS, Reshef R, Patel KP, Colligon TA, et al. (2009) Functional unresponsiveness and replicative senescence of myeloid leukemia antigenspecific CD8+ T cells after allogeneic stem cell transplantation. Clin Cancer Res 15: 4944-4953.

22. van Besien K, Artz A, Smith S, Cao D, Rich S, et al. (2005) Fludarabine, melphalan, and alemtuzumab conditioning in adults with standard-risk advanced acute myeloid leukemia and myelodysplastic syndrome. J Clin Oncol 23: 5728-5738.

23. van Besien K, Stock W, Rich E, Odenike O, Godley LA, et al. (2012) Phase I-II Study of Clofarabine-Melphalan-Alemtuzumab Conditioning for Allogeneic Hematopoietic Cell Transplantation. Biol Blood Marrow Transplant 18: 913921.

24. Kröger N, Bornhäuser M, Ehninger G, Schwerdtfeger R, Biersack $H$, et al. (2003) Allogeneic stem cell transplantation after a fludarabine/busulfan-based reduced-intensity conditioning in patients with myelodysplastic syndrome or secondary acute myeloid leukemia. Ann Hematol 82: 336-342.

25. Lim ZY, Ingram W, Brand R, Akthari M, Milojkovic D, et al. (2007) Clonal gammopathies following alemtuzumab-based reduced intensity conditioning haematopoietic stem cell transplantation: association with chronic graftversus-host disease and improved overall survival. Bone Marrow Transplant 40: $747-752$

\section{Acknowledgements}

The author would like to thank Dr. Stephanie Tsai for the careful editing and productive comments during the preparation of the manuscript. Dr. Hongtao Liu is supported by Cancer Research Foundation-Young Investigator Grant. 